\title{
Warum werden Frauen so selten MINT-Fachkräfte? Zur Bedeutung der Differenz zwischen mathematischen Kompetenzen und Selbstkonzept
}

Ben Jann und Sandra Hupka-Brunner, Universität Bern

Um den Fachkräftemangel im MINT-Bereich zu bekämpfen, bemühen sich Bund und Kantone, auf allen Bildungsstufen Interesse an Mathematik, Informatik, Naturwissenschaften und Technik zu wecken. Unter anderem soll dabei geschlechtsspezifischen Unterschieden hinsichtlich der Lehrstellen- bzw. Fächerwahl entgegengewirkt werden. Der vorliegende Beitrag setzt hier an und analysiert anhand der Daten der "ÜGK 2016", wie viele Jugendliche am Ende der obligatorischen Schulzeit denken, dass sie im Alter von 30 Jahren einen Beruf im Bereich der MINT-Fachkräfte ausüben werden. Dabei wird deutlich, dass bei jungen Frauen die Unterschätzung ihrer mathematischen Kompetenzen dazu beiträgt, dass sie sich seltener eine berufliche Zukunft im MINT-Bereich vorstellen können als junge Männer.

\section{Einleitung}

Aufgrund der veränderten Struktur des Schweizer Arbeitsmarktes ist seit etlichen Jahren ein erhöhter Bedarf an gut qualifizierten Arbeitnehmerinnen und Arbeitnehmern im MINT-Bereich ${ }^{1}$ feststellbar (Gardiol \& Gehrig, 2010). So stellt der Bundesrat (2010, S. 29) fest, dass sich seit den 1950er-Jahren der Anteil der MINT-Fachkräfte am Total der Erwerbstätigen fast verzehnfacht hat. Dieser gestiegenen Nachfrage stehen zwar auch vermehrt Absolventen und Absolventinnen aus MINT-Ausbildungen gegenüber, allerdings nicht in einem genügenden Ausmass, so dass es eine steigende Anzahl an Stellen im MINT-Bereich gibt, die nicht besetzt werden können. Dieser Fachkräftemangel droht sich aufgrund demographischer Entwicklungen in den nächsten Jahren $\mathrm{zu}$ verschärfen ${ }^{2}$ und ist vor allem in den Bereichen Informatik, Technik und teilweise auch im Bauwesen ausgeprägt.

Der Bundesrat hat in seinem Bericht von 2010 verschiedene Massnahmen zur Abschwächung des Fachkräftemangels im MINT-Bereich diskutiert und geht davon aus, dass dem Interesse an Mathematik sowie den fachlichen Selbst- 
konzepten (und in etwas geringerem Ausmass den fachlichen Kompetenzen selbst) eine zentrale Bedeutung bei der Wahl eines MINT-Studienfaches bzw. MINT-Ausbildungsberufes zukommt. Dementsprechend ist es dem Bund und den Kantonen ein Anliegen, auf allen Bildungsstufen das Interesse in den Bildungsfeldern Mathematik, Informatik, Naturwissenschaften und Technik zu fördern (Schweizerische Konferenz der kantonalen Erziehungsdirektoren [EDK], 2015). ${ }^{3}$ Insbesondere die seit Jahren persistenten Unterschiede zwischen den Geschlechtern hinsichtlich der Lehrstellen- bzw. Fächerwahl (OECD, 2012) sollen dabei angegangen werden. Ziel ist, Massnahmen zu erarbeiten, die dazu führen, dass die Berufs- und Studienwahl vermehrt von den Kompetenzen und dem Potenzial der Jugendlichen sowie von der Situation am Arbeitsmarkt und weniger von Geschlechter-Stereotypen und sozio-kultureller Herkunft geprägt ist. Angesichts der Tatsache, dass der schweizerische Arbeitsmarkt in immer stärkerem Masse Qualifikationen auf Tertiär-Niveau nachfragt (Meyer, 2016), scheint uns dieses Ziel vor allem auch in Hinblick auf die Studienfachfrage bedeutsam. Allerdings zeigt sich, dass die bisherigen Fördermassnahmen im MINT-Bereich kaum ausreichend sind (Leitungsgruppe MINT 2017; Petschick et al., 2013).

Der vorliegende Beitrag setzt hier an und analysiert anhand der Daten der ÜGK ${ }^{4}$ 2016, ob sich Jugendliche am Ende der obligatorischen Schulzeit vorstellen können, mit 30 Jahren in einem MINT-Beruf zu arbeiten, und wie diese Vorstellungen mit den fachlichen Selbstkonzepten der Jugendlichen sowie ihren Mathematikkompetenzen zusammenhängen. Im Fokus steht die Frage, welche mathematischen Selbstkonzepte junge Männer und Frauen im MINTBereich berichten und inwiefern diese Selbstkonzepte mit den tatsächlich erreichten Mathematikkompetenzen übereinstimmen. Schliesslich geht es darum zu klären, inwieweit eine systematische geschlechtsspezifische Diskrepanz zwischen Selbsteinschätzung und Kompetenzen dazu führt, dass Frauen ihre berufliche Zukunft sehr viel seltener im Bereich der MINT-Fachkräfte sehen als Männer.

\section{Theoretischer Rahmen und Forschungsstand}

\section{Erklärungsansätze und internationale Befunde}

Geschlechterunterschiede im MINT-Bereich werden international bereits seit Jahrzehnten diskutiert, wobei sowohl Kompetenzen als auch Selbstkonzepte von jungen Männern und Frauen im Zentrum stehen. In ihrem Überblick identifizieren Kriesi und Imdorf (2019) verschiedene theoretische Ansätze, die Gendersegregation in Bildungsverläufen erklären sollen.

Eine lange Tradition haben Ansätze, die davon ausgehen, dass sich die Geschlechterunterschiede in Ausbildungsverläufen durch rationale Entscheidungen erklären lassen. Die Grundannahme ist hier, dass der Nutzen verschie- 
dener Ausbildungsoptionen für Jungen und Mädchen unterschiedlich bewertet wird und diese wahrgenommenen Nutzendifferenzen zu gender-typischen Ausbildungsentscheidungen führen. Als Begründung werden zum einen geschlechtsspezifische Rollenbilder hinsichtlich Arbeitsmarkt und Familie herangezogen, die Jugendliche im Rahmen ihrer Abwägungen mit einbeziehen. ${ }^{5}$ Zum anderen wird angenommen, dass geschlechtsspezifische Unterschiede in den vermuteten Kompetenzen der Jugendlichen dazu führen, dass junge Männer eher Fächer im MINT-Bereich wählen. Aufgrund vermeintlich besserer Eignung junger Männer für ein entsprechendes Studium wird die Erfolgswahrscheinlichkeit als grösser eingeschätzt und die antizipierten Kosten, zum Beispiel in Form von notwendigen Anstrengungen, sind geringer. Kriesi und Imdorf (2019) weisen darauf hin, dass für diese zweite Argumentationslinie relativ wenig empirische Evidenz existiert. Allerdings könnten auch relative Kompetenzdifferenzen («comparative advantage thesis») zu gendertypischen Ausbildungswahlen führen, weil Jugendliche verschiedene Ausbildungsoptionen kompetenzbereichübergreifend evaluieren (Jonsson, 1999). Bei gleichen Mathematikkompetenzen, so die Argumentation, haben junge Frauen tendenziell Vorteile im sprachlichen Bereich, weshalb sie sich häufiger gegen MINT-Berufe entscheiden als junge Männer, obwohl sie entsprechende Mathematikkompetenzen mitbrächten. Weiterhin betonen Barone et al. (2017), dass es einen Unterschied machen kann, ob Entscheidungen eher entlang heuristischer Kriterien oder aufgrund konkreter Information getroffen werden. So konnte in einem Feldexperiment an italienischen Schulen gezeigt werden, dass die Ausbildungsentscheidungen weniger gendertypisch erfolgten, wenn junge Frauen gezielt über den Nutzen verschiedener Ausbildungsoptionen informiert wurden. Inwiefern diese Ergebnisse auf das schweizerische Berufsbildungssystem übertragbar sind, in dem der Berufswahlunterricht sehr gut verankert ist und die berufliche Orientierung bereits recht früh reflektiert wird, bleibt offen.

Eine zweite Familie von theoretischen Erklärungsansätzen nimmt eher eine kulturalistische Perspektive ein (Kriesi \& Imdorf, 2019), bei der vor allem Annahmen darüber im Zentrum stehen, welche Eigenschaften, Kompetenzen oder Verhaltensweisen für ein Geschlecht angemessen erscheinen. Dabei werden oftmals vorangegangene Sozialisationsprozesse dafür verantwortlich gemacht, dass geschlechtsspezifische Interessen, Selbstkonzepte und Werte zu unterschiedlichen Ausbildungsentscheiden führen. $\mathrm{Zu}$ diesen Ansätzen zählen etwa die Arbeiten von Correll (2004; siehe auch Ridgeway \& Corell, 2004), die betonen, dass geschlechtstypische Wahrnehmungen von Fähigkeiten zu unterschiedlichen Aspirationen und Entscheidungen führen können. Zentral ist hierbei, dass junge Männer und Frauen Erfolge unterschiedlich attribuieren und soziale FeedbackProzesse eine grosse Rolle spielen. Gemäss Kriesi und Imdorf (2019) gibt es verschiedene empirische Hinweise, die diese Sichtweise stützen. So zeigen etwa Sax et al. (2015), dass das mathematische Selbstkonzept einer der Schlüsselfaktoren für die Erklärung von Gender-Unterschieden im MINT-Bereich ist. Auch 
Eccles (1980, 2005) betont die Bedeutung von Sozialisationsprozessen, die (mit) dafür verantwortlich seien, dass sich junge Frauen - selbst wenn sie entsprechende Kompetenzen mitbringen - gegen MINT-Fächer entscheiden würden, weil junge Männer und Frauen je unterschiedliche Selbstkonzepte entwickelten. Eccles geht davon aus, dass junge Frauen seltener MINT-Fächer wählen, weil sie diese als weniger wichtig und weniger nützlich für den weiteren Lebensweg einschätzen und annehmen, dass das Studium solch eines Faches ihnen weniger Freude bereiten würde. Gemäss Kriesi und Imdorf (2019) wurde die Bedeutung von Werten und Interessen mehrfach bestätigt. Schliesslich verweisen auch Gottfredson und Lapan (1997) auf die Bedeutung von Gendernormen, die im Rahmen der Sozialisation erworben werden. Sie betonen, dass die Berufswahl als "circumscription and compromise» erfolge, bei der die Jugendlichen versuchen, akzeptable Optionen zu finden, bei denen ihr (soziales) Selbstkonzept sowohl zum Prestige als auch der Gendertypik des angestrebten Berufes passe. Auch für diese Sichtweise finden Kriesi und Imdorf (2019) empirische Unterstützung.

$\mathrm{Zu}$ den kulturalistisch ausgerichteten Erklärungsansätzen sind auch jene zu zählen, die geschlechtstypische Ausbildungsentscheidungen als Ausdruck aktueller Genderidentität sehen. So gehen etwa Charles und Bradley (2009) davon aus, dass die nach wie vor sehr hohe Gendersegregation im Ausbildungsbereich in den meisten westlichen Ländern dadurch zu erklären ist, dass es sich Jugendliche in individualisierten westlichen Gesellschaften leisten könnten, ihre Gender-Identität als Richtlinie der Ausbildungswahl zu nehmen, weil die meisten Ausbildungssysteme entsprechenden Handlungsspielraum für gendertypische Entscheidungen zulassen. Zudem kann angenommen werden, dass eine geschlechter-konforme Ausbildungswahl mit sozialer Anerkennung einhergeht, was insbesondere in der Jugendzeit von besonderer Bedeutung sein dürfte. Kriesi und Imdorf (2019) sehen dies in einigen Studien bestätigt, wobei sich nicht für alle Jugendlichen vergleichbare Effektmuster zeigen. Sie betonen, dass der bisherige Forschungsstand in dieser Richtung auf die Notwendigkeit und Bedeutung einer Intersektionalitätsperspektive verweise (siehe z.B. Gottburgsen \& Gross, 2012). Schliesslich betonen kulturalistisch ausgerichtete Erklärungsansätze auch die Bedeutung von Gatekeepern, (z.B. Lehrerinnen und Lehrern), die ihr Verhalten gegenüber den Jugendlichen an gesellschaftlichen Geschlechternormen ausrichten und somit Selbstkonzepte und Ausbildungsentscheidungen entsprechend mitprägen.

Insgesamt zeigt sich ein Konsens in der internationalen Forschung, dass den Selbstkonzepten von Jugendlichen eine zentrale Stellung bei der Wahl von MINT-Fächern zukommt. Ein grosser Teil der Literatur bezieht sich dabei auf Fächer- oder Studienwahlen in schulischen Bildungssystemen. Inwiefern sich dies auch auf die Situation in der Schweiz übertragen lässt, bleibt zu prüfen, da das schweizerische Bildungssystem durch einen hohen Anteil an dualen Berufslehren und einen verhältnismässig geringen Anteil an Tertiärbildung geprägt ist (SKBF, 2018). Im Folgenden soll daher dargestellt werden, wie sich Kompe- 
tenzen und Selbstkonzept in der Schweiz ${ }^{6}$ über die verschiedenen Schulstufen hinweg entwickeln und welchen Einfluss sie gemäss bestehendem Forschungsstand auf die beruflichen Aspirationen von Jugendlichen haben.

\section{Schweizer Forschungsstand zu MINT-Kompetenzen und Aspirationen}

Betrachtet man die Kompetenzentwicklung im Grundschulalter, zeigt sich, dass sich Gender-Unterschiede in Interessen, Selbstkonzepten und der Selbsteinschätzung eigener Fähigkeiten bereits früh abzeichnen (Bos et al., 2012; Valentin \& Jena, 1998). Hinsichtlich kognitiver Fähigkeiten in den verschiedenen Kompetenzbereichen sind die Unterschiede zwischen den Geschlechtern in diesem Alter jedoch eher gering (wobei eindeutige Befunde dadurch erschwert werden, dass bislang lediglich kantonale Daten vorliegen; SKBF, 2018). Am Ende der obligatorischen Schulzeit zeigt sich ein klareres Bild: Jungen haben international ${ }^{7}$ wie auch in der Schweiz ${ }^{8}$ bessere Kompetenzen in Mathematik und Naturwissenschaften, Mädchen weisen bessere Kompetenzen im Bereich Lesen auf. Diese Differenzen sind sehr stabil und seit der 1. PISA-Testung im Jahr 2000 nachweisbar. Bezüglich Mathematik scheinen die Differenzen allerdings eher im mittleren und oberen Leistungsbereich relevant zu sein, da in der ÜGK 2016 kaum Unterschiede zwischen den Geschlechtern in der Erreichung der mathematischen Grundkompetenzen gefunden wurden (Konsortium ÜGK, 2019).9

Auf der Sekundarstufe II kann zwischen der Berufsfindung (Lehrberuf) und der Wahl des Schwerpunktfachs am Gymnasium unterschieden werden. Dabei scheint die fachliche Segregation im Berufsbildungsbereich stärker ausgeprägt zu sein als im schulischen Bildungsbereich (Heiniger \& Imdorf 2018; Imdorf at al., 2015; Kriesi \& Imdorf 2019; Schwiter et al., 2014), was u.a. mit dem biographisch frühen Zeitpunkt der Entscheidung sowie der Selektion durch Lehrbetriebe zusammenhängen mag. Mathematik-Kompetenzen haben allerdings sowohl für Wahl eines MINT-Schwerpunktes im schulischen Bereich wie auch für die Berufswahl eine zentrale Bedeutung (Tomasik et al., 2018). Ein weiterer Befund ist, dass junge Männer häufig eine "männertypische» Wahl treffen, während junge Frauen in stärkerem Ausmass auch geschlechtsneutrale Berufe und Fächer anstreben (Tomasik et al., 2018). In Hinblick auf das Schwerpunktfach im Gymnasium lässt sich festhalten, dass der Anteil der Jugendlichen, die ein MINT-Schwerpunktfach wählen, zwischen den Kantonen stark schwankt, im gesamtschweizerischen Schnitt aber bei ca. 30\% liegt. Über die Zeit hinweg erweisen sich die kantonalen Anteile der Jugendlichen mit MINT-Schwerpunkt als recht stabil, es ist jedoch zu vermuten, dass sich die Anteile nicht immer mit dem Ausmass des Interesses der Jugendlichen an diesen Fächern decken, sondern auch eine Folge des institutionellen Angebots sind (SKBF, 2018). ${ }^{10}$ Junge Frauen sind in den Schwerpunktfächern Sprachen und Pädagogik, Psychologie und Künste deutlich übervertreten, junge Männer bei den Schwerpunktfächern Physik und Mathematik (SKBF, 2018). Gemäss 
SEATS (SKBF, 2018) existiert dabei ein deutlicher Zusammenhang zwischen individuellen Mathematikkompetenzen am Ende der obligatorischen Schulzeit und der Wahl eines MINT-Schwerpunktfachs. Bedeutsam scheint auch eine bei Mädchen im Schnitt stärker ausgeprägte Ablehnung von Wettbewerbsverhalten zu sein: So konnten Buser et al. (2017) nachzeichnen, dass im Kanton Bern MINT-Schwerpunktfächer am Gymnasium eher gewählt werden, wenn eine hohe Wettbewerbsbereitschaft vorliegt. Zudem erweist sich die Wahl eines MINT-Schwerpunktfaches als wichtiger Prädiktor für die Aufnahme eines entsprechenden MINT-Fachs im Studium. Jugendliche, die am Ende der obligatorischen Schulzeit hohe Mathematikkompetenzen vorweisen können, haben also eine höhere Wahrscheinlichkeit, ein MINT-Schwerpunktfach zu wählen. Dies wiederum erhöht die Wahrscheinlichkeit, ein Studium im MINT-Bereich aufzunehmen.

Vergleicht man im Tertiärbereich den Anteil der Studierenden im MINTBereich mit anderen Ländern, so weist die Schweiz einen durchschnittlichen Wert auf, allerdings ist der Frauenanteil in der Schweiz besonders gering (SKBF, 2018). Auch wenn die Geschlechterverteilung unter den Studierenden an der Universität insgesamt annähernd ausgeglichen ist, zeigt sich eine starke fachliche Geschlechtersegregation: Während junge Frauen in den exakten Wissenschaften untervertreten sind, sind junge Männer in den Sprachen, Literatur- und den Sozialwissenschaften, Veterinärmedizin und Pharmazie weniger präsent (SKBF, 2018). Diese starke horizontale Segregation erweist sich zudem als zeitlich sehr stabil. Die Situation an Fachhochschulen stellt sich sehr ähnlich dar, allerdings ist die horizontale Segregation noch ausgeprägter (SKBF, 2018), was einerseits am Fächerangebot mit weniger "geschlechtsneutralen» Fächern liegen könnte, andererseits an den vorgelagerten Bildungsetappen, die oftmals bereits Geschlechterungleichheiten aufweisen. Auch im Verlauf des Studiums scheinen zudem Geschlechterunterschiede zuzunehmen: Während sich beim Übergang vom Bachelor zum Master noch keine geschlechtsspezifischen Unterschiede in den Übertrittsraten ins Masterstudium zeigen, haben männliche Studierende nach dem Masterstudium höhere Übergangsquoten in weiterführende Ausbildungen wie das Doktorat, was zur vertikalen Segregation beiträgt (SKBF, 2018). Als Hauptfaktoren für die Wahl eines MINT-Faches (im gesamten Tertiärbereich) konnten Hupka-Brunner et al. (2012) das mathematische Interesse und Selbstkonzept sowie die allgemeine Selbstwirksamkeit identifizieren.

\section{Hypothesen}

Zusammenfassend zeigt sich für die Schweiz ein eher lückenhafter und fragmentierter Forschungsstand. Deutlich wird allerdings, dass Kompetenzunterschiede (vor allem in Mathematik) zu Beginn der Bildungslaufbahn nur gering sind, im Laufe der Zeit aber grösser werden. Unterschiede in den fachlichen Selbstkonzepten hingegen scheinen bereits früher ausgeprägt zu sein. Um einen Beitrag zum Schweizer Forschungsstand zu leisten, werden wir nachfolgend anhand 
eines aktuellen Datensatzes die MINT-Aspirationen der Jugendlichen quantifizieren und das Zusammenspiel zwischen mathematischen Kompetenzen und Selbstkonzept analysieren. Aufgrund der bisherigen Literatur ist davon auszugehen, dass beide Aspekte für die Wahl eines MINT-Berufes relevant sind, die Selbsteinschätzungen aber unter Umständen sogar eine wichtigere Rolle spielen als die tatsächlichen Kompetenzen. So konnten etwa Hofer und Stern (2016) für die Schweiz darlegen, dass selbst mathematisch sehr begabte Mädchen Physik und Mathematik aufgrund ihres Selbstkonzeptes und mangelndem Interesse abwählen. ${ }^{11}$ Weiterhin weisen die schon sehr früh ausgeprägten Geschlechterdifferenzen in den Selbstkonzepten darauf hin, dass diese einem starken Einfluss gesellschaftlicher Normen und von Gender-Stereotypen ausgesetzt sind.

Es geht uns nicht einfach nur darum zu zeigen, inwieweit geschlechtsspezifische Unterschiede in den Kompetenzen und Selbsteinschätzungen je einen Beitrag zur Erklärung des Gender-Gaps in den MINT-Aspirationen leisten. Wir interessieren uns spezifisch dafür, inwieweit die Diskrepanz zwischen Kompetenzen und Selbsteinschätzungen eine geschlechtsspezifische Komponente aufweist, die für die Wahl eines MINT-Berufes relevant ist. Entsprechend formulieren wir die folgenden Hypothesen:

H1: Weibliche Jugendliche sehen ibre berufliche Zukunft deutlich seltener im Bereich der MINT-Fachkräfte als männliche Jugendliche.

H2: Es gibt mehr weibliche als männliche Jugendliche, die trotz hoher Mathematikkompetenzen ein eher geringes mathematisches Selbstkonzept aufweisen.

H3: Jugendliche mit geringem mathematischen Selbstkonzept sehen ihre eigene berufliche Zukunft selbst bei hohen Mathematikkompetenzen weniger häufig im Bereich der MINT-Fachkräfte als Jugendliche mit hohem mathematischen Selbstkonzept.

Aus der Kombination von $\mathrm{H} 1$ bis $\mathrm{H} 3$ folgt zudem:

H4: Ein Teil des Geschlechterunterschieds in den MINT-Aspirationen ergibt sich dadurch, dass weibliche Jugendliche auch bei vergleichbaren mathematischen Kompetenzen ein systematisch geringes mathematisches Selbstkonzept aufweisen als männliche Jugendliche.

\section{Daten und Operationalisierung}

Die folgenden Analysen ${ }^{12}$ beruhen auf den Daten der Studie zur Überprüfung des Erreichens der Grundkompetenzen aus dem Jahr 2016 (Konsortium ÜGK, 2016). ${ }^{13}$ Im Rahmen der Studie wurden rund 22’000 Schülerinnen und Schüler der Schweiz am Ende der obligatorischen Schule (im letzten Quartal der 9. - bzw. 
nach HarmoS-Zählung 11. - Klasse) im Fach Mathematik geprüft und u.a. zu ihrem familiären Hintergrund und ihren Zukunftsplänen befragt. ${ }^{14}$ Sämtliche Analysen erfolgen unter Berücksichtigung des Survey-Designs anhand der im Datensatz bereitgestellten BRR-Gewichte («balanced repeated replication»). Die Operationalisierung der zentralen Variablen gestaltet sich wie folgt:

Berufliche Zukunft im MINT-Bereich: Die Antizipation der eigenen beruflichen Zukunft im MINT-Bereich der Schülerinnen und Schüler wurde in der ÜGK 2016 anhand der Frage "Was meinst du, welchen Beruf wirst du mit 30 Jahren haben?» erhoben und liegt u.a. in ISCO-08-Codierung (International Standard Classification of Occupations) vor. Für die Zuteilung der Berufe zum Bereich der MINT-Fachkräfte orientieren wir uns an der Klassifikation von Gehrig et al. (2010). Tabelle A1 im Anhang gibt eine Übersicht über die von Gehrig et al. (2010) vorgeschlagenen Kategorien und die von uns zugeteilten ISCO-08Berufsgruppen. In der Tabelle ausgewiesen sind zudem die Prozentanteile der verschiedenen Kategorien nach Geschlecht.

Anhand der dargestellten Klassifikation bilden wir zwei binäre MINTIndikatoren: einen Indikator für MINT-Berufe im engeren Sinne, dessen Kategorien in der Tabelle mit einem Stern markiert sind, und einen Indikator für MINT-Berufe im weiteren Sinne. ${ }^{15}$ Weiterhin berücksichtigen wir in den Analysen nur diejenigen Personen, für die eine gültige Berufskodierung vorliegt. Wir schliessen somit rund 25\% der Personen aus, weil sie die Frage nach der beruflichen Zukunft nicht beantwortet haben. Bei diesen Fällen handelt es sich grösstenteils um Personen, die keine konkrete Vorstellung über ihren Beruf mit 30 haben.

Fachkompetenz in Mathematik: Die Kompetenzen in Mathematik übernehmen wir aus den Leistungstests der ÜGK. Aus Gründen der Einfachheit verwenden wird den WLE-Score ("weighted likelihood estimate») und arbeiten nicht mit den «Plausible Values».

Selbsteinschätzung der Mathematik-Kompetenz: Dazu verwenden wir zwei alternative Operationalisierungen. Einerseits eine Skala zum mathematischen Selbstkonzept, die aus den Items "Im Fach Mathematik bekomme ich gute Noten", "Mathematik ist eins meiner besten Fächer», "Ich war schon immer gut in Mathematik» gebildet wurde (wir greifen hier auf die in den ÜGK-Daten bereitgestellte Skalierung zurück) und widerspiegelt, welches Bild die Jugendlichen von sich als Schüler/in in Mathematik haben; andererseits eine Skala zur mathematischen Selbstwirksamkeit, die auf Angaben dazu beruht, wie sicher sich die befragte Person ist, gegebene mathematische Aufgaben lösen zu können, sich also auf konkrete mathematische Probleme bezieht und nicht auf ein generalisiertes Selbstbild. In den ÜGK-Daten liegt diese Skala in Form von vier Subskalen vor (Rechnen, Algebra, Geometrie, Wahrscheinlichkeiten), die wir als einfaches arithmetisches Mittel zu einer Gesamtskala zusammenfassen. 
Geschlecht: Für das Geschlecht liegen in den Daten zwei Angaben vor, einerseits das Geschlecht gemäss Stichprobenrahmen und andererseits das selbstberichtete Geschlecht. Wir verwenden die selbstberichtete Angabe. ${ }^{16}$

Wie bereits erwähnt, schränken wir die nachfolgenden Analysen auf Schülerinnen und Schüler ein, für die eine gültige Angabe zum Beruf mit 30 vorliegt (knapp 75\% der Personen). Weiterhin schliessen wir einige wenige Beobachtungen aus, die einen fehlenden Wert für die mathematische Fachkompetenz, das mathematische Selbstkonzept, die mathematische Selbstwirksamkeit, oder das Geschlecht aufweisen. Die verbleibende Anzahl Beobachtungen für die Analyse beträgt $N=16116$.

\section{Ergebnisse}

Erwartungsgemäss besteht hinsichtlich MINT-Aspirationen eine ausgeprägte Geschlechterdifferenz (Hypothese 1). Abbildung 1 zeigt den Anteil von Männern und Frauen, deren erwarteter Beruf mit 30 dem Bereich der MINT-Fachkräfte zugeordnet werden kann (unter denjenigen Personen, für die eine gültige Angabe zum Beruf mit 30 vorliegt). Nach enger MINT-Definition streben 19.0\% der männlichen Jugendlichen einen MINT-Beruf an; nach erweiterter Definition erhöht sich dieser Wert auf ein Viertel (24.7\%). Bei den weiblichen Jugendlichen beträgt der MINT-Anteil nach enger Definition nur gerade 2.7\%, nach erweiterter Definition sind es 7.9\%. Die beruflichen Vorstellungen der befragten Jugendlichen weisen hinsichtlich der Präferenz für einen Beruf aus dem Bereich der MINT-Fachkräfte also einen äusserst ausgeprägten Gender-Bias auf.

Wie kann eine solche Diskrepanz erklärt werden? Gemäss Hypothese 2 schätzen weibliche Jugendliche ihre mathematischen Fähigkeiten durchschnittlich als geringer ein als männliche Jugendliche, selbst wenn sich die tatsächlichen fachlichen Kompetenzen nicht unterscheiden. Gemäss Hypothese 3 führt ein tiefes mathematisches Selbstkonzept zu einer geringeren Wahrscheinlichkeit, die eigene berufliche Zukunft in einen MINT-Beruf zu antizipieren. Zusammengenommen, so Hypothese 4, sollte also zumindest ein Teil der Geschlechterdifferenz dadurch zu erklären sein, dass Frauen ihre Mathematikkompetenzen eher unterschätzen als Männer (bzw. Männer ihre Kompetenzen eher überschätzen).

Evidenz zu Hypothese 2 findet sich in Abbildung 2, in der die Über- und Unterschätzung der eigenen Kompetenzen nach Geschlecht dargestellt ist. Da die Skalen der Selbsteinschätzung und die Ergebnisse der Leistungstests nicht direkt vergleichbar sind, greifen wir auf Differenzen in Rängen zurück. Das heisst, wir generieren für jede Person den relativen Rang in der Verteilung der Leistungstests wie auch die Ränge in den Verteilungen der Selbsteinschätzungsskalen, und bilden dann die Differenzen der Ränge. Eine negative Differenz bedeutet, dass die Person in der Selbsteinschätzung einen tieferen Rang belegt als in den Leistungstests (und ihre Leistungen somit relativ zu den anderen Personen 


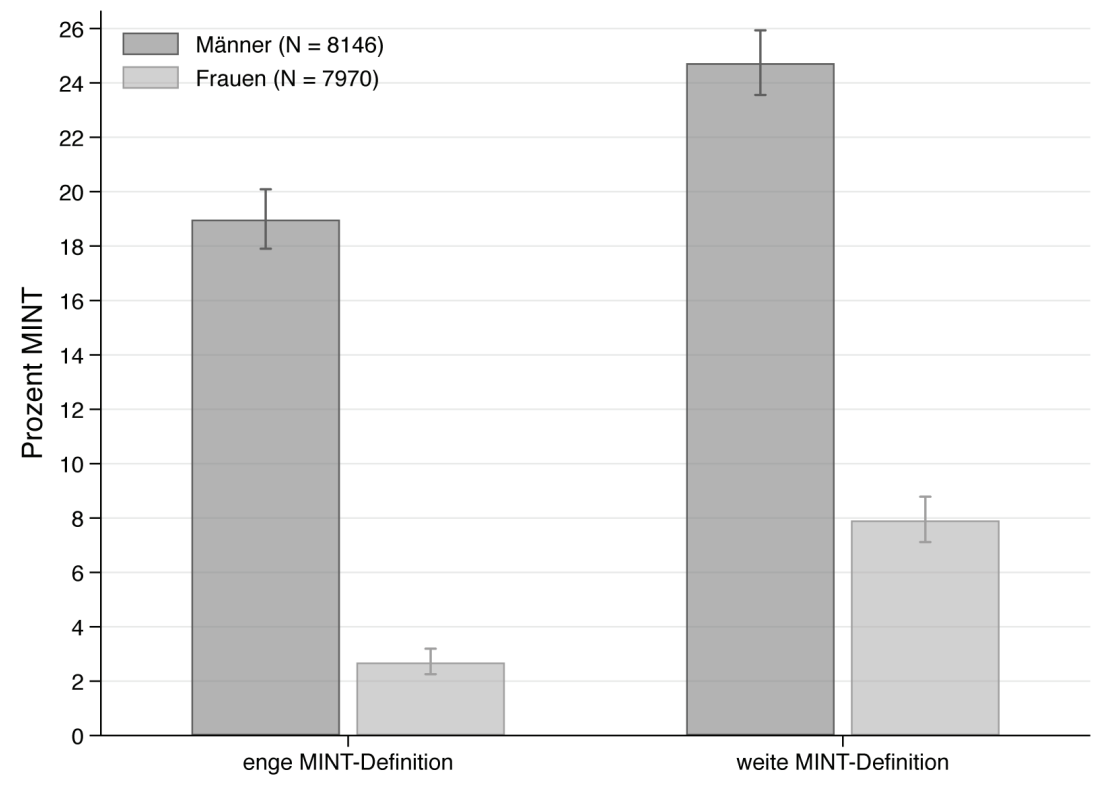

Abb. 1. Anteil «Beruf mit 30» im Bereich der MINT-Fachkräfte nach Geschlecht

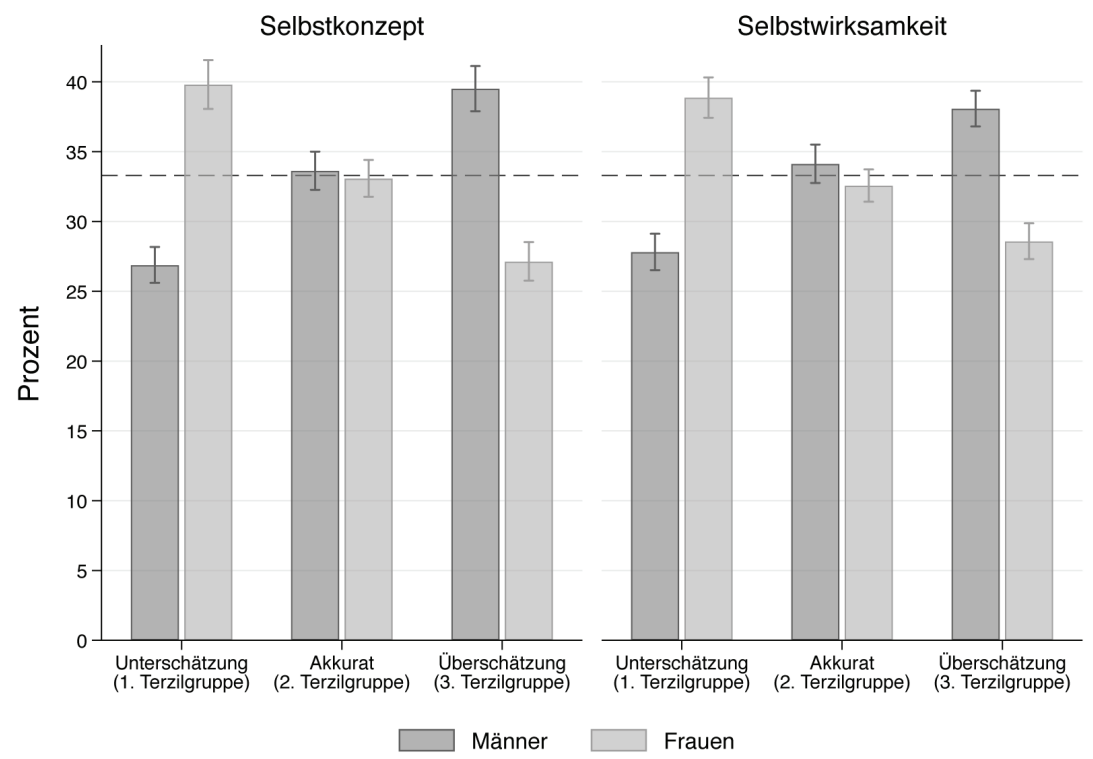

Abb. 2. Verteilung von Männern und Frauen über die Terzilgruppen der Rangdifferenz zwischen Selbsteinschätzung und Kompetenzmessung 
eher unterschätzt), eine positive Differenz kommt einer relativen Überschätzung der eigenen Kompetenzen gleich. Zur Veranschaulichung der Resultate fassen wir die Personen in Abbildung 2 zu Gruppen zusammen. Konkret unterteilen wir die Personen anhand der Terzile der Rangdifferenzen in drei gleich grosse Gruppen: (1) ein Drittel Personen, deren Rangdifferenzen am stärksten negativ sind (i.e., die sich am stärksten unterschätzten), (2) ein Drittel Personen mit mittleren Rangdifferenzen, (3) ein Drittel Personen, die sich am stärksten überschätzten. Sodann berechnen wir, wie sich die weiblichen und männlichen Jugendlichen über die drei Gruppen verteilen. Falls sich Frauen und Männer in ihren Selbsteinschätzungen nicht systematisch unterscheiden, sollte sich in jeder Gruppe jeweils ein Drittel der Frauen bzw. ein Drittel der Männer befinden. In Abbildung 2 sieht man jedoch, dass die empirischen Ergebnisse stark von der Gleichverteilung abweichen: Frauen sind in der 1. Gruppe (Unterschätzung) deutlich übervertreten und in der 3. Gruppe (Überschätzung) deutlich untervertreten; bei den Männern sind die Verhältnisse entsprechend umgekehrt.

Der Zusammenhang gilt für beide Skalen, das mathematischen Selbstkonzept wie auch die mathematische Selbstwirksamkeit, scheint aber bei letzterer etwas weniger stark ausgeprägt zu sein. Dies wird auch durch eine Analyse der Mittelwertdifferenzen der Rangdifferenzen nach Geschlecht bestätigt. Beim Selbstkonzept beträgt die Mittelwertdifferenz 11.3 Rangpunkte (95\%-Konfidenzintervall: 10.0 bis 12.5), bei der Selbstwirksamkeit hingegen nur 7.1 Rangpunkte (95\%-Konfidenzintervall: 6.1 bis 8.0). ${ }^{17}$ Der Befund eines schwächeren Geschlechtereffekts bei der Selbstwirksamkeit erstaunt wenig, da die Skala der Selbstwirksamkeit auf Fragen zu konkreten Rechenaufgaben beruht, während sich die Skala des Selbstkonzepts auf eher allgemeine Einschätzungen bezieht, die sich wahrscheinlich einfacher durch geschlechtsspezifische Rollenvorstellungen beeinflussen lassen. So scheint die Skala der Selbstwirksamkeit denn auch generell zutreffendere Einschätzungen der eigenen Fähigkeiten zu liefern als die Skala des Selbstkonzepts: für erstere Skala erreicht die Korrelation mit den Leistungstests einen Wert von 0.60, bei letzterer liegt dieser Wert nur bei 0.35. ${ }^{18}$ Trotz dieses Unterschieds, das sei nochmals betont, finden wird bei beiden Skalen einen starken und hoch signifikanten Gender-Bias.

Gemäss Hypothese 3 führt eine tiefe Selbsteinschätzung der eigenen Mathematikkompetenzen zu einer geringeren Antizipation eines Berufes im MINTBereich, selbst wenn für Kompetenzen kontrolliert wird. Tabelle 1 zeigt Ergebnisse von logistischen Regressionen, bei denen die Wahl eines MINT-Berufes mit 30 auf den Rang im Leistungstest sowie die Rangdifferenz zwischen Selbsteinschätzung und Leistungstest regrediert wird. Dargestellt sind durchschnittliche Marginaleffekte, die als Differenzen in Wahrscheinlichkeiten interpretiert werden können. Ein Koeffizient von 1 bedeutet, dass eine Erhöhung im Leistungstest bzw. der Rangdifferenz um einen Rangpunkt mit einer Erhöhung der Wahrscheinlichkeit eines MINT-Berufs um einen Prozentpunkt einhergeht. Man erkennt, dass die Selbsteinschätzung einen starken und hochsignifikanten 
eigenständigen Zusammenhang mit dem MINT-Beruf aufweist, wobei der Effekt beim (allgemeinen) Selbstkonzept wiederum etwas stärker ausfällt als bei der (spezifischen) Selbstwirksamkeit. Beispielsweise geht bei der engen MINTDefinition eine Überschätzung (Unterschätzung) der eigenen Kompetenzen um einen Rangpunkt mit einer Erhöhung (Verringerung) der MINT-Wahrscheinlichkeit um 0.19 Prozentpunkte einher, wenn das mathematsche Selbstkonzept zur Bildung der Rangdifferenzen zugrunde gelegt wird. Bei der mathematischen Selbstwirksamkeit ist der entsprechende Effekt etwas schwächer und beträgt 0.15 Prozentpunkte.

Tab. 1. Wahrscheinlichkeit eines "Berufs mit 30" im Bereich der MINT-Fachkräfte in Abhängigkeit der Ergebnisse des Leistungstests und der Selbsteinschätzung

\begin{tabular}{lcccc}
\hline & \multicolumn{2}{c}{ Enge MINT-Definition } & \multicolumn{2}{c}{ Weite MINT-Definition } \\
\hline & $\begin{array}{c}\text { Selbst- } \\
\text { konzept }\end{array}$ & $\begin{array}{c}\text { Selbst- } \\
\text { wirksamkeit }\end{array}$ & $\begin{array}{c}\text { Selbst- } \\
\text { konzept }\end{array}$ & $\begin{array}{c}\text { Selbst- } \\
\text { wirksamkeit }\end{array}$ \\
\hline Rang im Leistungstest & 0.299 & 0.243 & 0.383 & 0.335 \\
& $(22.0)$ & $(18.3)$ & $(25.1)$ & $(21.3)$ \\
Rangdifferenz Selbsteinschätzung & 0.190 & 0.151 & 0.194 & 0.176 \\
& $(16.8)$ & $(11.1)$ & $(15.8)$ & $(11.4)$ \\
\hline
\end{tabular}

Anmerkungen: Durchschnittliche Marginaleffekte basierend auf logistischen Regressionen, t-Werte in Klammern, $N=16116$.

Aus der Kombination der bisherigen Resultate (weibliche Jugendliche haben geringere MINT-Aspirationen; weibliche Jugendliche unterschätzen ihre Mathematikkompetenzen eher; eine Unterschätzung der Mathematikkompetenzen führt zu einer Verringerung der MINT-Aspirationen) folgt, dass ein Teil der Geschlechterdifferenz in der Wahl von MINT-Berufen auf die unterschiedliche Selbsteinschätzung zurückzuführen sein wird (Hypothese 4). Wir prüfen dies anhand einer Zerlegung der Geschlechterdifferenz in den MINT-Präferenzen in die Erklärungsbeiträge durch die Leistungs- und Selbsteinschätzungsvariablen (wiederum unter Verwendung von Rängen bzw. Rangdifferenzen). ${ }^{19}$ Tabelle 2 zeigt die Ergebnisse der Zerlegung, getrennt nach enger sowie weiter MINTDefinition, wobei die Erklärungsbeiträge als prozentuale Anteile an der gesamthaften Geschlechterdifferenz ausgedrückt sind (wie in Abbildung 1 dargestellt, haben Frauen nach enger Definition eine um 16.3 Prozentpunkte tiefere Wahrscheinlichkeit, einen MINT-Beruf zu wählen, nach weiter Definition beträgt die Differenz 16.8 Prozentpunkte).

Frauen weisen in den Leistungstests etwas tiefere Werte auf als Männer (um durchschnittlich 5.2 Rangpunkte; 95\%-Konfidenzintervall: 4.1 bis 6.3), und bessere Mathematikleistungen gehen mit einer höheren MINT-Präferenz einher. Ein Teil der Geschlechterdifferenz in den MINT-Präferenzen wird deshalb durch Leistungsunterschiede erklärt: Bei der engen MINT-Definition sind dies 11.8\%, bei der weiten Definition 13.8\%. Über die Leistungsdifferenzen hinaus trägt aber auch die verzerrte Selbsteinschätzung der eigenen Kompetenzen substanziell 
zum Geschlechtereffekt bei. In den Dekompositionen berücksichtigen wir beide Rangdifferenzen simultan. Zusammengenommen erklären die Fehleinschätzungen $14.4 \%$ bzw. $13.7 \%$ des Geschlechterunterschieds, wobei - wie aufgrund der bisherigen Resultate zu erwarten - der Einfluss des mathematischen Selbstkonzepts stärker ausfällt als der Einfluss der mathematischen Selbstwirksamkeit (Erklärungsanteile von 11.5 vs. $2.9 \%$ bei enger MINT-Definition bzw. 9.6 vs. $4.1 \%$ bei weiter Definition). ${ }^{20}$

Tab. 2. Prozentuale Erklärungsbeiträge der Leistungs- und Selbsteinschätzungsvariablen an die Geschlechterdifferenz in der Wahrscheinlichkeit eines "Berufs mit 30» im Bereich der MINT-Fachkräfte

\begin{tabular}{lcccc}
\hline & \multicolumn{2}{c}{ Enge MINT-Definition } & \multicolumn{2}{c}{ Weite MINT-Definition } \\
\hline Leistungstest & 11.8 & $(9.4 ; 14.2)$ & 13.8 & $(11.2 ; 16.5)$ \\
Selbstkonzept & 11.5 & $(8.8 ; 14.3)$ & 9.6 & $(7.0 ; 12.2)$ \\
Selbstwirksamkeit & 2.9 & $(1.1 ; 4.7)$ & 4.1 & $(2.1 ; 6.0)$ \\
Selbstkonzept + Selbstwirksamkeit & 14.4 & $(11.5 ; 17.4)$ & 13.7 & $(10.7 ; 16.6)$ \\
\hline Total & 26.2 & $(22.7 ; 29.8)$ & 27.5 & $(24.1 ; 30.8)$ \\
\hline
\end{tabular}

Anmerkungen: Ergebnisse von Oaxaca-Blinder-Dekompositionen mit Logit-Link und zusammengefasstem Referenzmodell (siehe Fussnote 19), 95\%-Konfidenzintervalle in Klammern, $N=16116$.

Insgesamt erklären die berücksichtigten Leistungs- und Einstellungsvariablen gut ein Viertel der Geschlechterdifferenz in der Wahrscheinlichkeit, dass der «Beruf mit 30» im Bereich der MINT-Fachkräfte liegt (26.2\% bei enger MINT-Definition; 27.5\% bei weiter Definition). Dieser Erklärungsbeitrag ist substanziell, bedeutet allerdings gleichzeitig, dass ein Grossteil des Unterschieds zwischen weiblichen und männlichen Jugendlichen hinsichtlich der Präferenz für einen MINT-Beruf auf weitere Faktoren wie beispielsweise allgemeine Vorstellungen über rollenadäquate Tätigkeiten für Frauen und Männer zurückzuführen sein dürfte. Trotzdem liefern unsere Ergebnisse Hinweise auf einen möglichen Mechanismus, der zumindest für einen Teil der geschlechtsspezifischen Berufswahl verantwortlich ist. Kann der Gender-Bias in der Bewertung der eigenen Mathematikkompetenzen abgebaut werden, würden wir aufgrund dieser Ergebnisse erwarten, dass sich die Präferenzen von weiblichen und männlichen Jugendlichen für einen MINT-Beruf etwas angleichen. Darüber hinaus dürfte ein Feedback-Mechanismus in dem Sinne wirksam sein, dass der Gender-Bias hinsichtlich mathematischer Fähigkeiten bzw. die Vorstellung über eine unterschiedliche «Begabung» von Frauen und Männern für Mathematik auch für die Unterschiede in den Leistungstests mitverantwortlich ist. Wir würden also erwarten, dass sich durch eine Reduktion dieses Gender-Bias auch die tatsächlichen Leistungen angleichen, was eine weitere Verringerung der Unterschiede in den MINT-Präferenzen zur Folge hätte. 


\section{Diskussion / Fazit}

Warum werden Frauen so selten MINT-Fachkräfte? Für dieses international viel diskutierte Thema lagen bislang in der Schweiz nur wenige Ergebnisse vor. Unsere Analysen haben gezeigt, dass junge Frauen ibre Mathematik-Kompetenzen öfter unterschätzen als junge Männer und dass diese Unterschätzung der eigenen Fachkompetenz dazu führt, dass junge Frauen ihre berufliche Zukunft seltener im MINT-Bereich sehen als junge Männer - selbst dann, wenn sie die entsprechenden Kompetenzen dafür mitbringen. Möchte man also mehr Frauen für den MINT-Bereich gewinnen, müsste die Stärkung der fachlichen Selbstkonzepte junger Frauen mitberücksichtigt werden, so dass sie zu realistischeren Einschätzungen ihrer Kompetenzen gelangen. Welche Rolle dabei die konkreten Unterrichtsprozesse oder die Benotungspraktiken der Lehrkräfte (Hofer, 2015) spielen, bleibt zu diskutieren.

Darüber hinaus ist zum Beispiel gemäss Eccles (2005) sowie Gottfredson und Lapan (1997) davon auszugehen, dass Kompetenzen und Selbstkonzepte in MINT-Fächern eine wichtige, aber keine ausreichende Bedingung für die Wahl von MINT-Berufen darstellen. Wie bereits erwähnt, kann die Wahl von Ausbildungsoptionen durch den Wert des Fachs für den antizipierten Lebensweg beeinflusst werden (Eccles, 2005). Da nach wie vor mehr Mädchen als Jungen die Vereinbarkeit von Familie und Beruf ins Zentrum ihrer Ausbildungswahl stellen (Kanji \& Hupka-Brunner, 2015; Wehner et al., 2016), und dabei eine erschwerte Vereinbarkeit in «typisch männlichen» (zumeist MINT-) Berufen antizipieren (Schwiter et al., 2014), weist ein MINT-Beruf einen geringeren Wert für den Lebensentwurf weiblicher Jugendlicher auf. Männliche Jugendliche hingegen knüpfen ihre Vaterrolle immer noch häufig an das Prinzip des männlichen Haupternährers und streben entsprechend gut bezahlte berufliche Positionen an (Schwiter et al., 2014). Zentral scheinen in diesem Zusammenhang auch familiäre Vorbilder und Rollen-Stereotype zu sein (Kahn \& Ginther, 2017). So fanden Schwiter et al. (2014) Hinweise, dass Jugendliche in geschlechteruntypischen Berufen oftmals innerhalb der Familie berufliche Vorbilder hatten, das heisst, dass diese Jugendlichen häufig einen untypischen Beruf wählten, den ihre Eltern oder andere nahe Bezugspersonen ausübten. Ergänzende Analysen im Rahmen dieses Beitrags (nicht dargestellt) haben gezeigt, dass sich eine MINTVererbung innerhalb der Familie nachweisen lässt, allerdings nur für die Väter: Haben Jugendliche einen Vater, der im MINT-Bereich arbeitet, erhöht dies die Wahrscheinlichkeit, dass sie selber ihre berufliche Zukunft in diesem Bereich antizipieren. Ob sie diese dann auch tatsächlich realisieren, bliebe mittels der Daten der TREE-Folgewellen, bei denen u.a. der jeweilige Ausbildungsstatus erhoben wird, zu analysieren. Vielversprechend scheint für die weitere Forschung zudem auch der Intersektionalitätsansatz (Gottburgsen \& Gross, 2012), bei dem geprüft wird, ob und inwiefern die Kreuzung verschiedener Ungleichheitsdimensionen (z.B. soziale Herkunft, Migrationshintergrund, Geschlecht) zu unterschiedlichen Leistungen, Selbstkonzepten und Aspirationen führt. 
Die Untervertretung von Frauen in MINT-Berufen auf dem Arbeitsmarkt speist sich allerdings nicht nur aus der horizontalen Segregation der vorgelagerten Bildungsetappen (Marti \& Bertschy, 2013), sondern scheint sich auch dadurch zu verschärfen, dass junge Frauen aus MINT-Berufen häufiger aussteigen als ihre männlichen Kollegen (SKBF, 2018), was unter Umständen mit einem MINTspezifischen, wettbewerbs- und wenig familienfreundlichen Arbeitsklima erklärt werden kann, welches Frauen die Vereinbarkeit von Familie und Beruf erschwert (Kahn \& Ginther, 2017). Möchte man in Zeiten des Fachkräftemangels im MINT-Bereich also vermehrt junge Frauen gewinnen, sind nicht nur bildungspolitische, sondern auch arbeitsmarktpolitische Massnahmen in den Blick zu nehmen.

\section{Anmerkungen}

1 MINT steht für Mathematik, Informatik, Naturwissenschaften und Technik.

2 Die aktuelle Altersstruktur der Erwerbsbevölkerung legt nahe, dass in den nächsten 10 bis 20 Jahren vermehrt MINT-Fachkräfte aus dem Arbeitsmarkt ausscheiden werden. Zeitgleich ist davon auszugehen, dass die Studierendenzahlen aufgrund der geringeren Geburtenraten der nachfolgenden Generationen dies nicht auszugleichen vermögen.

3 Bereits in der Primarschule wird versucht, Interesse am MINT-Bereich zu wecken und Kinder für naturwissenschaftliche und technische Themen zu interessieren, u.a. durch Lektionen im Bereich «Mensch, Natur, Umwelt».

4 Überprüfung des Erreichens der Grundkompetenzen (http://uegk-schweiz.ch/).

5 Gemäss diesem Ansatz sehen sich junge Männer nach wie vor in der Rolle des Familienernährers und richten ihre berufliche Laufbahn eher am Einkommen aus, wohingegen junge Frauen die Vereinbarkeit zwischen Familie und Beruf antizipieren und berufliche Optionen höher werten, die eine bessere Vereinbarkeit versprechen. Gemäss Kriesi und Imdorf (2019) konnten auf internationaler Ebene verschiedene Studien diese Sichtweise stützen. Auch für die Schweiz gibt es Hinweise in dieser Richtung (Kanji \& HupkaBrunner, 2015; Schwiter et al., 2014; Wehner et al., 2016).

6 Wobei wir teilweise auch Ergebnisse aus Deutschland referieren, wenn keine Überblicksarbeiten für die Schweiz vorliegen, da die Bildungssysteme relativ ähnlich sind.

7 International gesehen zeigt sich, dass Jungen in den meisten, aber nicht allen Ländern höhere naturwissenschaftliche Kompetenzen sowie ein grösseres Interesse an MINTFächern haben (Mostafa, 2019).

8 Wobei die Schweizer Jugendlichen gemäss PISA im Bereich Mathematik und Naturwissenschaften insgesamt deutlich bessere Leistungen erzielen als die Jugendlichen der Nachbarländer (SKBF, 2018).

9 Hingegen konnte ein starker Einfluss der sozialen Herkunft festgestellt werden, und auch der Migrationshintergrund hat einen bedeutsamen Einfluss auf das Erreichen der Grundkompetenzen. Interessanterweise zeigen sich zudem kantonale Differenzen in der Wirkungsweise dieser Einflussfaktoren (Konsortium ÜGK, 2019).

10 Gestützt wird diese Annahme u.a. dadurch, dass in jenen Kantonen mit geringem MINTSchwerpunktfach-Anteil die Studierenden, die ein MINT-Studium beginnen, überproportional aus anderen Schwerpunktfächern stammen.

11 Wobei die Autoren das mangelnde Interesse auf die konkrete Unterrichts-Situation beziehen und nicht auf das Fach an sich. Das geringere Selbstkonzept der Mädchen könnte zudem auch (wenn auch nicht ausschliesslich) in Zusammenhang mit dem Verhalten von Lehrkräften stehen. So konnte Hofer (2015) zeigen, dass Lehrerinnen und Lehrer der Sekundarstufe I, die seit weniger als zehn Jahren unterrichten, Mädchen im Fach Physik 
bei gleichen Leistungen signifikant schlechter benoten als Jungen, was Auswirkungen auf das Selbstkonzept der jungen Mädchen haben und ihre Erfolgserwartungen in diesem Fach mindern dürfte. Auch international deuten Befunde auf Diskriminierungsprozesse bei der Notengebung hin (Terrier, 2015).

12 Eine vollständige Dokumentation der Analysen ist unter http://dx.doi.org/10.7892/ boris. 134223 verfügbar.

13 Nidegger, C. (2019). ÜGK / COFO / VECOF 2016. Competencies of Swiss pupils in mathematics [Dataset]. FORS. https://doi.org/10.23662/FORS-DS-1004-1

14 Dabei diente die ÜGK 2016 als Baseline für die 2. TREE-Kohorte (siehe auch https:// www.tree.unibe.ch/). TREE war massgeblich an der Entwicklung der Erhebungsinstrumente und der Durchführung der ÜGK 2016 beteiligt.

15 Die Unterscheidung wurde von Gehrig et al. (2010) übernommen. Bei den MINT-Berufe i.e.S. ist die Zuordnung zum MINT-Bereich unserer Ansicht nach unstrittig, während die weite Definition zusätzliche Berufe enthält, bei denen die Zuordnung weniger eindeutig erscheint (und die weniger stark von Fachkräftemangel betroffen sind; siehe Gehrig et al., 2010, S. 8).

16 Die Abweichungen zwischen den beiden Angaben sind nur minimal. In unserem Analysesample von 16116 Personen stimmt die Geschlechtsangabe in 65 Fällen nicht überein.

17 Zur Einordnung: Der theoretische Skalenbereich der Rangdifferenzen erstreckt sich von -100 bis 100 . Weiterhin beträgt die mittlere absolute Rangdifferenz 26.1 Punkte beim Selbstkonzept und 19.9 Punkte bei der Selbstwirksamkeit. Auch wenn wir diese unterschiedliche Variation berücksichtigen, erscheint der Geschlechtereffekt beim Selbstkonzept stärker (43.1\% der mittleren absoluten Rangdifferenz) als bei der Selbstwirksamkeit (35.5\%).

18 Es besteht eine Korrelation von 0.48 zwischen den beiden Selbsteinschätzungsskalen. Bei den berichteten Werten handelt es sich um Rangkorrelationen; die linearen Korrelationen weichen nur marginal davon ab.

19 Es handelt sich um eine sogenannte Oaxaca-Blinder-Dekomposition (Jann, 2008), wobei wir aufgrund des binären Charakters der abhängigen Variablen logistische Regressionen zur Modellierung verwenden und die Geschlechterdifferenz gemäss Vorschlag von Yun (2004) zerlegen. Als Referenzmodell dient ein zusammengefasstes Modell über beide Geschlechter, in dem das Geschlecht als zusätzlicher Prädiktor enthalten ist (zur Begründung einer solchen Modellierung siehe Jann, 2008). Ergänzend haben wir erweiterte Dekompositionen unter Berücksichtigung einer Reihe von Kontrollvariablen durchgeführt (siehe die Analysedokumentation unter http://dx.doi.org/10.7892/boris.134223). Die Resultate für die in Tabelle 2 aufgeführten Variablen ändern sich dadurch nur marginal. Bei den berücksichtigten Kontrollvariablen handelt es sich um Indikatoren dafür, ob die Berufe der Eltern im Bereich der MINT-Fachkräfte liegen, ob die Person Nachhilfeunterricht in Mathematik erhalten hat, die Schulnoten in Mathematik, ob die Person eine gymnasiale Schulstufe besucht, ob die Person Klassen wiederholt hat, eine Skala für Absentismus, der Migrationsstatus, der sozio-ökonomische Status der Familie (höchster ISEI von Vater und Mutter), sowie die Anzahl Bücher im Haushalt. Zusammengenommen leisten die Kontrollvariablen keinen signifikanten Erklärungsbeitrag, und auch auf Ebene der einzelnen Variablen finden sich kaum systematische Resultate. Einzig für den Besuch einer gymnasialen Schulstufe ist ein signifikanter, wenn auch nur schwach ausgeprägter Erklärungsbeitrag zu verzeichnen (ca. 1\% der Geschlechterdifferenz; der Erklärungsbeitrag kommt dadurch zustande, dass Frauen öfter das Gymnasium besuchen und mit dem Besuch des Gymnasiums die Wahrscheinlichkeit eines MINT-Berufs tendenziell abnimmt). Für die anderen Variablen finden sich zwar teilweise substanzielle Effekte auf die Wahrscheinlichkeit eines MINT-Berufs (z.B. ist eine MINT-Vererbung in dem Sinne zu beobachten, dass Kinder von Vätern im MINT-Bereich eher davon ausgehen, selbst in 
Zukunft einen MINT-Beruf auszuüben), die weiblichen und männlichen Jugendlichen unterscheiden sich jedoch kaum hinsichtlich dieser Merkmale. Ferner finden wir auch keine Hinweise auf unterschiedliche Wirkmechanismen zwischen den Geschlechtern, d.h. es bestehen keine signifikanten Unterschiede zwischen weiblichen und männlichen Jugendlichen hinsichtlich der Effekte der Variablen auf die MINT-Wahrscheinlichkeit (dies gilt auch für die Leistungs- und Selbsteinschätzungsvariablen).

20 Die Effekte für die Mütter sind nicht signifikant, was auch an der geringen Fallzahl von Müttern, die im MINT-Bereich erwerbstätig sind, liegen könnte.

\section{Literatur}

Barone, C., Schizzerotto, A., Abbiati, G. M., \& Assirelli, G. (2017). Gender, information barriers and fields of study choice: a field experiment (LIEPP Working Paper No. 63). Laboratory for Interdisciplinary Evaluation of Public Policies. http://spire.sciencespo.fr/ hdl:/2441/6nemhd9te89l1akhtlfqe8589f

Bos, W., Wendt, H., Köller, O., \& Selter, C. (Hrsg.). (2012). TIMSS 2011. Mathematische und naturwissenschaftliche Kompetenzen von Grundschulkindern in Deutschland im internationalen Vergleich. Waxman.

Bundesrat. (Hrsg.). (2010). Mangel an MINT-Fachkräften in der Schweiz. Ausmass und Ursachen des Fachkräftemangels in MINT (Mathematik, Informatik, Naturwissenschaften und Technik). Bericht des Bundesrates https:/www.sbfi.admin.ch/dam/sbfi/de/ dokumente/webshop/2010/mangel_an_mint-fachkraefteninderschweiz.pdf

Buser, T., Peter, N., \& Wolter, S. (2017). Gender, Willingness to Compete and Career Choices along the Whole Ability Distribution (IZA Discussion Paper Series No. 10976). IZA Institute of Labor Economics. http://ftp.iza.org/dp10976.pdf

Charles, M., \& Bradley, K. (2009). Indulging our gendered selves? Sex segregation by field of study in 44 Countries. American Journal of Sociology, 114, 927-976.

Corell, S. (2004). Constraints into preferences: Gender, status, and emerging career aspirations. American Sociological Review, 69, 93-113.

Eccles, J. S. (1980). Final report. Self perception, task perception and academic choice: origins and change (NIE Grant NIE-G-78-0022). University of Michigan

Eccles, J. S. (2005). Studying gender and ethnic differences in participation in Math, Physical Science, and Information Technology. New Directions for Child and Adolescent Development, 110, 7-14.

EDK. (Schweizerische Konferenz der kantonalen Erziehungsdirektoren). (2015). Chancen optimal nutzen. Erklärung 2015 zu den gemeinsamen bildungspolitischen Zielen für den Bildungsraum Schweiz. https://edudoc.ch/record/117294

Gardiol, L., \& Gehrig, M. (2010). Der MINT-Fachkräftemangel - Ausmass, Ursachen und Auswirkungen. Die Volkswirtschaft, 9, 52-55.

Gehrig, M., Gardiol, L., \& Schaerrer, M. (2010). Der MINT-Fachkräftemangel in der Schweiz. Ausmass, Prognose, konjunkturelle Abhängigkeit, Ursachen und Auswirkungen des Fachkräftemangels in den Bereichen Mathematik, Informatik, Naturwissenschaften, Technik. Staatssekretariat für Bildung und Forschung SBF.

Gottburgsen, A., \& Gross, C. (2012). Welchen Beitrag leistet «Intersektionalität» zur Klärung von Kompetenzunterschieden bei Jugendlichen? In R. Becker, \& H. Solga (Hrsg.). Soziologische Bildungsforschung. Sonderheft 52 der Kölner Zeitschrift für Soziologie und Sozialpsychologie (S. 86-110). Springer VS.

Gottfredson, L. S., \& Lapan, R. T. (1997). Assessing gender-based circumscription of occupational aspirations. Journal of Career Assessment, 5, 419-441.

Heiniger, M., \& Imdorf, C. (2018). The role of vocational education in the transmission of gender segregation from education to employment: Switzerland and Bulgaria compared. Journal for Labour Market Research, 52(15), 1-21. https://doi.org/10.1186/s12651-018-0248-6 
Hofer, S. I. (2015). Studying gender bias in physics grading: the role of teaching experience and country. International Journal of Science Education, 37, 2879-2905.

Hofer, S. I., \& Stern, E. (2016). Underachievement in physics: When intelligent girls fail. Learning and Individual Differences, 51, 110-139.

Hupka-Brunner, S., Kanji, S., Bergman, M. M., \& Meyer, T. (2012). Gender differences in the transition from secondary to post-secondary education in Switzerland (Report commissioned by the OECD [TOR/PISA-1]). Universität Basel. https://www.tree.unibe. ch/e206328/e305140/e305154/files305285/Hupka-Kanji-Bergman-Meyer_2012_

Switzerland_OECD_ReportFinal_en_ger.pdf

Imdorf, C., Hegna, K., \& Reisel, L. (Eds.). (2015). Gender segregation in vocational education. Emerald.

Jann, B. (2008). The Blinder-Oaxaca decomposition for linear regression models. The Stata Journal, 8, 453-479.

Jonsson, J. O. (1999). Explaining sex differences in occupational choice: An empirical assessment of a rational choice model. European Sociological Review, 15, 391-404.

Kahn, S., \& Ginther, D. (2017). Women and STEM (NBER Working Paper No. 23525). http://www.nber.org/papers/w23525

Kanji, S., \& Hupka-Brunner, S. (2015). Young women's strong preference for children ans subsequent occupational gender segregation. What is the link? Equality, Diversity and Inclusion: An International Journal, 34, 124-140.

Konsortium ÜGK. (Hrsg.). (2019). Überprüfung der Grundkompetenzen. Nationaler Bericht der ÜGK 2016: Mathematik 11. Schuljahr. EDK und SRED. https://10.18747/ PHSG-coll3/id/386

Kriesi, I., \& Imdorf, C. (2019). Gender Segregation in Education. In R. Becker, (Ed.), Research Handbook on Sociology of Education (S. 193-212). Edward Elgar Publishing.

Leitungsgruppe MINT. (2017). Schlussbericht Mandat MINT 2013-2016 http:// www.akademien-schweiz.ch/index/Foerderung-MINT/mainColumnParagraphs/03/ download_website.pdf

Marti, M., \& Bertschy, K. (2013). BELODIS - Berufseinstieg und Lohndiskriminierung neue Erklärungsansätze zu einer Schlüsselphase für geschlechtsspezifische Ungleichheiten. (Zusammenfassung der Projektergebnisse) http://www.nfp60.ch/SiteCollectionDocuments/nfp60_marti_zusammenfassung_projektergebnisse_lang.pdf

Meyer, T. (2016). Bildungsgrenzen im Spiegel der Panel-Studie TREE. TREE. https://10.7892/ boris. 130989

Mostafa, T. (2019). Why don't more girls choose to pursue a science career? PISA in Focus, 93 https://10.1787/02bd2b68-en

OECD. (2012). Gender Equality in Education, Employment and Entrepreneurship: Final Report to the MCM 2012. OECD. https://www.oecd.org/employment/50423364.pdf

Petschick, G., Schmidt, R., \& Norkus, M. (2013). Frauenförderung zwischen heterogenen Logiken: Der Fall eines Nachwuchsförderungprogramms in der deutschen Exzellenzinitiative. Schweizerische Zeitschrift für Soziologie, 39, 383-404.

Ridgeway, C., \& Corell, S. (2004). Unpacking the gender system. A theoretical perspective on gender beliefs and social relations. Gender \& Society, 18, 510-531.

Sax, L. J., Kanny, M. A., \& Riggers-Piehl, T. A. (2015). «But I'm Not Good at Math»: The Changing Salience of Mathematical Self-Concept in Shaping Women's and Men's STEM Aspirations. Research in Higher Education, 56, 813-842.

Schwiter, K., Hupka-Brunner, S., Wehner, N., Huber, E., Kanji, D. S., Maihofer, A., \& Bergman, M. M. (2014). Warum sind Pflegefachmänner und Elektrikerinnen nach wie vor selten? Geschlechtersegregation in Ausbildungs- und Berufsverläufen junger Erwachsener in der Schweiz. Schweizerische Zeitschrift für Soziologie, 40, 401-428.

SKBF. (2018). Bildungsbericht Schweiz 2018. Schweizerische Koordinationsstelle für Bildungsforschung. 
Terrier, C. (2015). Giving a little help to girls? Evidence on grade discrimination and its effect on students achievement (CEP discussion paper No. 1341). Centre for Economic Performance, LSE. https://ideas.repec.org/p/cep/cepdps/dp1341.html

Tomasik, M. J., Oostlander, J., \& Moser, U. (2018). Von der Schule in den Beruf. Wege und Umwege in der nachobligatorischen Ausbildung. Bildungsdirektion Kanton Zürich.

Valentin, R., \& Jena, S. (1998). Geschlechtsspezifische Unterschiede im Grundschulalter Ergebnisse aus Forschungsprojekten. Frauen- und Geschlechterforschung in den Erziehungswissenschaften, 17, 3-12.

Wehner, N., Schwiter, K., Hupka-Brunner, S., \& Maihofer, A. (2016). Geschlechterungleichheiten in Ausbildungs-und Berufsverläufen junger Erwachsener in der Schweiz. Ergebnisse aus einer Mixed-Methods-Studie. In H. Faulstich-Wieland (Hrsg.), Berufsorientierung und Geschlecht (S. 23-38). Beltz Juventa.

Yun, M. (2004). Decomposing differences in the first moment. Economics Letters, 82, $275-280$.

Schlagworte: MINT, Gender, Mathematik-Kompetenzen, mathematisches Selbstkonzept, Berufswahl

\section{Pourquoi les femmes deviennent-elles si rarement des professionnelles des STIM ? L'importance de la différence entre les compétences mathématiques et l'image de soi}

\section{Résumé}

Pour lutter contre la pénurie de main-d'œuvre qualifiée dans le secteur des STIM (sciences, technologies, ingénierie et mathématiques), la Confédération et les cantons s'efforcent de susciter l'intérêt pour les STIM à tous les niveaux de l'enseignement. Cette mesure vise notamment à contrecarrer les différences entre les sexes lors du choix des apprentissages et des matières. Dans ce contexte, nous utilisons les données "ÜGK 2016» pour analyser la part de jeunes en fin de scolarité obligatoire qui pensent avoir un emploi dans le secteur des STIM à l'âge de 30 ans. Les résultats montrent clairement qu'en raison d'une sous-estimation de leurs propres compétences en mathématiques, les jeunes femmes sont moins susceptibles que les jeunes hommes d'imaginer une carrière dans le domaine des STIM.

Mots clés: Secteur des STIM, genre, compétences mathématiques, concept de soi mathématique, choix de carrière 


\section{Perché le donne diventano così raramente professioniste in STEM? L'importanza della differenza tra le competenze matematiche e il concetto di sé}

\section{Riassunto}

Per contrastare la carenza di manodopera qualificata nel settore STEM (scienza, tecnologia, ingegneria e matematica), la Confederazione e i Cantoni si sforzano di suscitare l'interesse per tale settore a tutti i livelli di formazione. Uno degli obiettivi è quello di contrastare le differenze specifiche di genere nella scelta dei posti di tirocinio e dei campi di studio. Questo articolo utilizza i dati dello studio VeCoF 2016 per analizzare quanti giovani alla fine della scuola dell'obbligo prospetta un lavoro nel settore delle STEM all'età di 30 anni. I risultati mostrano chiaramente che la sottovalutazione delle competenze matematiche delle giovani donne contribuisce a far sì che esse siano meno propense dei giovani uomini a immaginare un futuro professionale nel settore STEM.

Parole chiave: Settore della scienza, tecnologia, ingegneria e matematica, genere, competenze matematiche, concetto di sé matematico, scelte professionali

\section{Why do women so rarely become STEM professionals? The role of discrepancy between mathematics skills and self-concept}

\footnotetext{
Abstract

To counter the shortage of skilled workers in the fields of STEM, the federal government and the cantons try to arouse interest in science, technology, engineering, and mathematics at all levels of education. One of the aims is to counteract gender-specific differences in the choice of apprenticeships and fields of study. Against this backdrop, we use data from the "ÜGK 2016" to analyze how many young people at the end of compulsory schooling think that they will have a STEM profession at the age of 30. The results make clear that due to an underestimation of their own mathematical skills, young women are less likely than young men to imagine a career in the fields of STEM.
}

Keywords: STEM, gender, mathematics skills, mathematical self-concept, occupational choice 


\section{Anhang}

Tabelle A1: MINT-Kategorien und zugeordnete ISCO-08-Berufsgruppen

\begin{tabular}{rrr}
\hline MINT-Kategorie & Männer & Frauen \\
& $(N=$ & $(N=$ \\
$10740)$ & $10541)$ \\
\hline
\end{tabular}

Informatik

1.1* Informatik (Informatik, Informatik- und Softwareingenieurwesen, Wirtschafts- und Betriebsinformatik)

2500 Akademische und vergleichbare Fachkräfte in der Informations-

und Kommunikationstechnologie, onA

2511 Systemanalytiker

2512 Softwareentwickler

2513 Web- und Multimediaentwickler

2514 Anwendungsprogrammierer

2529 Akademische und vergleichbare Fachkräfte für Datenbanken

und Netzwerke, anderweitig nicht genannt

3514 Webmaster

\section{Technik}

1.2* Elektrotechnik (Elektrotechnik und Elektroingenieurwesen)

2151 Ingenieure im Bereich Elektrotechnik

3113 Elektrotechniker

1.3* Maschinentechnik (Maschinentechnik und Maschineningenieurwesen,

Aviatik, Automobiltechnik)

2144 Maschinenbauingenieure

3115 Maschinenbautechniker

1.4* Mikrotechnik (Elektronik und Elektroingenieurwesen, Mikrotechnik und

Mikrotechnikingenieurwesen, Systemtechnik, Mechatronik, Kommunikationssysteme, Telekommunikationstechnik)

2152 Ingenieure im Bereich Elektronik

3114 Techniker im Bereich Elektronik

3510 Techniker für den Betrieb von Informations- und Kommunika-

tionstechnologie und für die Anwenderbetreuung, onA

3521 Techniker für Rundfunk und audiovisuelle Medien

3522 Telekommunikationstechniker

1.5* Wirtschaftsingenieurwesen (Betriebs- und Produktionswissenschaften,

Wirtschaftsingenieurwesen, Medieningenieurwesen)

2141 Wirtschafts- und Produktionsingenieure

3122 Produktionsleiter bei der Herstellung von Waren

1.6* Anderes aus Technik \& IT (Materialwissenschaften, Holztechnik, Werkstoffe- und Materialingenieurwesen, andere/fachrichtungsübergreifende Richtungen aus Technik \& IT)

2140 Ingenieurwissenschaftler (ohne Elektrotechnik, Elektronik und Telekommunikation), onA

2149 Ingenieure, anderweitig nicht genannt

3110 Material- und ingenieurtechnische Fachkräfte, onA

3118 Technische Zeichner

3119 Material- und ingenieurtechnische Fachkräfte, anderweitig nicht genannt 
Bauwesen

2.1* Bau (Bauingenieurwesen, Gebäudetechnik, Heizungs-, Lüftungs- und

Klimaanlageningenieurwesen)

2142 Bauingenieure

3112 Bautechniker

3120 Produktionsleiter im Bergbau, bei der Herstellung von Waren und im Bau, onA

3123 Bauleiter

3132 Steuerer von Verbrennungs- und Wasserbehandlungsanlagen

2.2 Planung und Vermessung (Geomatik, Geodäsie, Vermessungsinge-

nieurwesen, Umweltingenieurwesen, Kulturechnik und Kulturingenieurwesen, Raum-, Landschafts-, Siedllungs- \& Ortsplanung)

2143 Umweltschutzingenieure

2160 Architekten, Raum-, Stadt- und Verkehrsplaner, Vermessungsingenieure und Designer, onA

2164 Raum-, Stadt- und Verkehrsplaner

2165 Kartografen und Vermessungsingenieure

2.3 Architektur

2161 Architekten

2162 Landschaftsarchitekten

2.4 Anderes aus Bauwesen (Andere / fachrichtungsübergreifende Richtungen aus dem Bauwesen)

\section{Chemie \& Life Sciences (C\&LS)}

3.1* Chemie (Chemie, Chemieingenieurwesen, Lebensmittelwissenschaften, Lebensmitteltechnologie, Verfahrenstechnik)

2113 Chemiker

2145 Chemieingenieure

3111 Chemo- und Physikotechniker

3116 Chemiebetriebs- und Verfahrenstechniker

3.2 Biotechnologie (Biotechnologie, Life Sciences, Molecular Life Sciences,

Life Science Technologies, Lebesnmittelwissenchaften, Lebensmitteltechnologie)

3141 Biotechniker (ohne medizinische Fachberufe)

3.3 Gesundheit (Pharmazie, Pharmatechnologie, Medizinaltechnik, Medizinaltechnologie)

3212 Medizintechniker im Bereich Labor und Pathologie

3213 Pharmazeutisch-technische Assistenten

3214 Medizinische und zahnmedizinische Prothetiktechniker

3255 Physiotherapeutische Techniker und Assistenten

3.4 Anderes aus C\&LS (Andere / fachrichtungsübergreifende Richtungen aus C\&LS)

\section{Andere MINT}

4.1 Geografie (Geografie, Umwelt(natur)wissenschaften, andere/

fachrichtungsübergreifende Richtungen aus den Geo- und Umweltwissenschaften)

2114 Geologen und Geophysiker

2131 Biologen, Botaniker, Zoologen und verwandte Berufe

2133 Umweltwissenschaftler 
4.2* Exakte Wissenschaften (Mathematik, Statistik, Physik, Astronomie, andere/fachrichtungsübergreifende exakte Wissenschaften)

2111 Physiker und Astronomen

2112 Meteorologen

2120 Mathematiker, Versicherungsmathematiker und Statistiker

4.3 Andere MINT (Agronomie, Forstwirtschaft, andere/kategorieübergreifende MINT-Fachrichtungen)

2132 Agrar-, Forst- und Fischereiwissenschaftler und -berater

3142 Agrartechniker

3143 Forsttechniker

\begin{tabular}{lrr}
\hline Sonstige Berufe & 56.40 & 68.93 \\
Weiss nicht, keine Antwort, nicht codierbar, etc. & 25.08 & 25.15 \\
\hline Total & 100.00 & 100.00 \\
\hline
\end{tabular}

Anmerkungen: Die MINT-Kategorien und deren Beschreibungen wurden aus Tabelle 2 in Gehrig et al. (2010) übernommen. Die Zuordnung der ISCO-08-Berufsgruppen stammt von uns. Häufigkeitsverteilungen und Berücksichtigung der Stichprobengewichte. Ausgeschlossen wurden Beobachtungen, denen die Frage nicht gestellt wurde (i.d.R. wegen Abbruch des Interviews) oder die für die anderen zentralen Variablen unserer Analyse (Geschlecht, Fachkompetenz in Mathematik, Selbsteinschätzung der Mathekompetenz) fehlende Werte aufweisen.

* MINT-Berufe nach enger Definition.

Ben Jann, Prof. Dr. Professor für Soziologie, insb. Sozialstrukturanalyse, an der Universität Bern. Hauptantragsteller des Infrastrukturprojekts TREE und Mitglied des Boards des Interfaculty Centre for Educational Research (ICER). Mitwirkung bei der Entwicklung des Kontextfragebogens der Überprüfung des Erreichens der Grundkompetenzen (ÜGK). Forschungsschwerpunkte: soziale Ungleichheit und Methoden der empirischen Sozialforschung.

Universität Bern, Institut für Soziologie, Fabrikstr. 8, CH-3012 Bern.

E-Mail: ben.jann@soz.unibe.ch

Sandra Hupka-Brunner, Dr. phil, Ko-Leiterin des Forschungsprojektes TREE - Transition von der Erstausbildung ins Erwerbsleben an der Uni Bern. Mitwirkung bei der Entwicklung des Kontextfragebogens der Überprüfung des Erreichens der Grundkompetenzen (ÜGK). Forschungsschwerpunkte: Transitionen im Bildungswesen und ins Erwerbsleben, Gender, Migration, soziale Ungleichheiten.

Universität Bern, Institut für Soziologie, Fabrikstr. 8, CH-3012 Bern.

E-Mail: sandra.hupka@soz.unibe.ch 